\title{
Binding Mechanism Analysis between AhR Protein and Typical Polybrominated Diphenyl Ether via Molecule Docking
}

\author{
Long Jiang a, Gaojun Liu ${ }^{\mathrm{b}}$, Qing $\mathrm{Li}^{\mathrm{c}}$ \\ North China Electric Power Research Institute Co Ltd. Beijing 100045, China. \\ akaveykikiy@163.com, bliu.gao.jun@163.com, ‘@ncepri@139.com
}

\begin{abstract}
In this paper, BDE-85 was selected as the typical polybrominated diphenyl ether (PBDE) because of its obvious biological toxicity and the receptor binding affinity (RBA) was taken as the index of biological toxicity. By using the homology modeling, the aryl hydrocarbon receptor (AhR) protein molecule was established for researching the binding mechanism between the AhR protein and BDE-85. The obtained results showed that: the RSMD value of the established AhR protein was 0.049 , present reasonable molecule structure and amino acid sequence; among active residues, the hydrophobic amino form a hydrophobic region to improve the molecule docking between the AhR protein and BDE-85, resulting in significant biological toxicity of BDE-85.
\end{abstract}

Keywords: Polybrominated diphenyl ethers; aryl hydrocarbon receptor; homology modeling; molecular docking.

\section{Introduction}

As a typical type of persistent organic pollutants (POPs), polybrominated diphenyl ethers (PBDEs) have been verified might have endocrine disrupting effects, producing significant negative effect on environment and health of organisms [1]. Previous studies have shown that the biological toxicity of PBDEs has a close connection with the (AhR), which can result in the dissociation of molecular chaperones and produce harm on organisms [2]. Meanwhile, the binding affinity of PBDEs to AhR (Receptor Binding Affinities, RBA) had been widely to evaluate the level of biological toxicity due to the positive correlation between them.

Molecular docking is a kind of method to carry out drug design via research the characteristics of the receptor and the interaction between the receptor and drug molecules, which has been validated as an important research technology in the field of computer aided drug design [3]. The aim of this paper is to research the binding mechanism between the AhR protein molecule and typical PBDE congener via molecule docking, which can provide theoretical guide for analyzing the biological toxicity of PBDEs.

\section{Methods}

\subsection{Homology modeling}

Because of no existing protein of AhR, the homology modeling was first used to construct the protein of AhR: the amino acid sequence of the AhR protein domain of mice (sequence number GI: 7304873, residues number: 276-384) was obtained from the National Center for Biotechnology Information (NCBI, http://www.ncbi.nlm.nih.gov/protein/7304873) [4]; The PAS protein domain of HIF-2R was queried as the template protein for homology modeling, then the amino acid sequence of the AhR protein and the template protein were submitted to the Automated Protein Modeling Server applied by the Glaxo Smith Kline to form the AhR protein via the homology modeling [5]. 


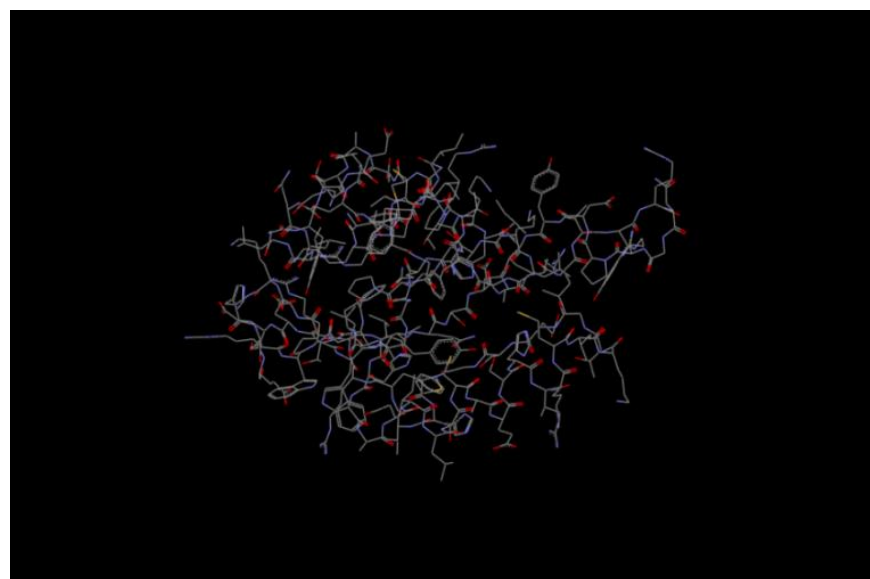

\subsection{Molecular docking}

Fig. 1 The molecule structure of the established AhR protein

The process of molecular docking and biological toxicity mechanism analysis is divided into the following 4 steps [6]:

(1)Ligand preparation

In this paper, we select BDE-85 as typical PBDEs because it's obvious biological toxicity. The optimal configuration of BDE-85 was calculated via Gaussian 09, which was loaded into Discovery Studio 2.5 software for further preparation: the Powell capability gradient optimization method; the CHARMm force field; the Gasteiger-Huckel charge; 1000 iterations; the energy convergence is limited to $0.01 \mathrm{~kJ} / \mathrm{mol}$.

(2)Molecular docking

With the help of Flexible-Docking module in Discovery Studio 2.5 software package, the AhR protein receptor binding cavity is determined and the flexible docking between the ligand protein and protein binding cavity formed via the integrate into of the ligand molecules (Fig. 2).

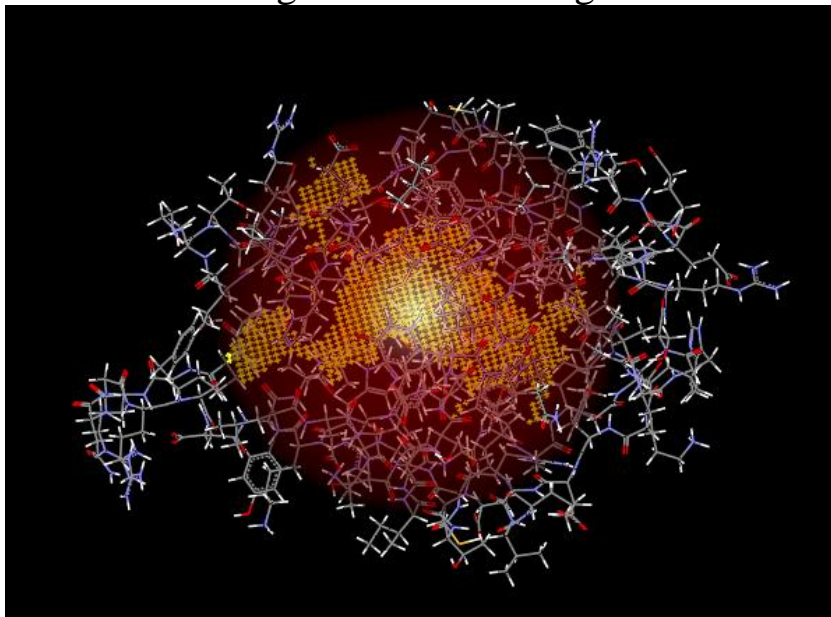

Fig. 2 The formed AhR protein receptor binding cavity via flexible docking

(3) Evaluation of molecular docking

The root mean square deviation (RMSD) between pre-docking and docking molecules was applied to evaluate the reasonable of the docking result. If the $\mathrm{RMSD} \leq 0.2 \mathrm{~nm}$, the docking model can simulate the actual the binding model between the ligand and receptor, and also prove the rationality of the original set parameters. In this paper, the RMSD of the docking between AhR and BDE-85 was 0.049 through the homology modeling.

\section{Results and Discussion}

When BDE-85 was bonded to AhR, the dominant amino acid residues were mainly distributed around BDE-85 in a certain distance, which can be identified as active residues. Fig 3 has shown the binding between BDE- 85 and the established AhR protein, the specific different interaction force 
descriptors involved in the molecule docking were shown in Table 1. As can be seen from Fig. 3, the interaction between the BDE-85 and AhR protein contain 2 categories: residues of THR283, HIS285, CYS327, SER330, SER340, GLY341, LEU348, SER369, GLN377 (colored with pink) play an important stabilizing effect on the binding by hydrogen bonding, electrostatic force (only SER369 and BDE-85 form hydrogen bond); residues of PHE289, ILE301, LEU309, PHE318, ILE319, HIS320, PHE345, LEU347, VAL357, GLN358 (colored with green) are mainly Van der Waals forces. Further form the view of the hydrophilic/hydrophobic of the amino acid (Table 2), the residues colored with pink (except LEU348) is hydrophobic groups, so it can conclude that the hydrophobic region formed by these hydrophobic amino acids can occur significant hydrophobic interaction with BDE-85. When BDE-85 (with hydrophobic) entered into receptor protein, the original protein molecule was destroyed and formed into cage structure, resulting in the increase of molecular system entropy, material together and the decrease of the energy of the whole system (AhR protein and BDE-85).

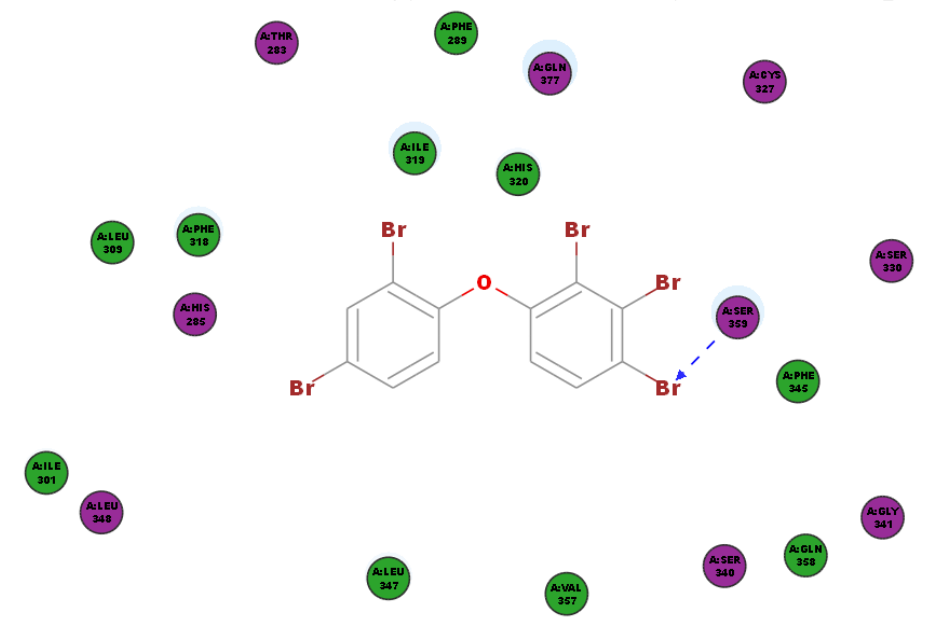

Fig. 3 The binding between BDE-85 and the established AhR protein

Table 1 Interaction force description of molecular docking

Introduction
Amino acids involved in hydrogen bonding, electrostatic or polar interactions
Amino acids involved in Van der Waals forces
Solvent effect: the radius of the circle is positive corresponding with the solvent
effect

Table 2 Hydrophilic/hydrophobic of the active residues

\begin{tabular}{cccc}
\hline Residues & Hydrophilic/hydrophobic & Residues & Hydrophilic/hydrophobic \\
\hline Cys & Hydrophilic & Gln & Hydrophilic \\
Phe & hydrophobic & Ser & Hydrophilic \\
His & Hydrophilic & Thr & Hydrophilic \\
Ile & hydrophobic & Val & hydrophobic \\
Leu & hydrophobic & Tyr & hydrophobic \\
\hline
\end{tabular}

\section{Summary}

(1) The established AhR protein via the homology modeling have been validated has reasonable amino acid sequence, with the RSMD (0.049) is less than $0.2 \mathrm{~nm}$, which can be used to carry out the molecule docking. 
(2) The active residues main contain two categories, and the hydrophobic amino acid residues play an important stabilizing effect on the binding by hydrogen bonding and electrostatic force by forming a hydrophobic region to improve the molecule docking.

\section{References}

[1] A. K. Peters, J. T. Sanderson, A. Bergman, M. van der Berg, Antagonism of TCDD-induced ethoxyresorufin-O-deethylation activity by polybrominated diphenyl ethers (PBDEs) in primary cynomolgus monkey (Macaca fascicularis) hepatocytes. Toxicol. Lett. 164 (2006) 123-132.

[2] S. Kovarich, E. Papa, P. Gramatica, QSAR classification models for the prediction of endocrine disrupting activity of brominated flame retardants. J. Hazard. Mater. 190 (2011) 106-112.

[3] F. Li, X. H. Li, X. L. Liu, Docking and 3D-QSAR studies on the Ah receptor binding affinities of polychlorinated biphenyls (PCBs), dibenzo-p-dioxins (PCDDs) and dibenzofurans (PCDFs). Environmental Toxicology and Pharmacology. 32 (2011) 478-485.

[4] W. H. Yang, X. H. Liu, Y. Wu, J. P. Giesy, H. X. Yu, Molecular docking and comparative molecular similarity indices analysis of estrogenicity of polybrominated diphenyl ethers and their analogues. Environmental Toxicology \& Chemistry. 29 (2010) 660 - 668.

[5] K. Arnold, L. Bordoli, J. Kopp, and The SWISS-MODEL Workspace: A web-based environment for protein structure homology modelling. Bioinformatics. 22 (2006) 195-201.

[6] A.N. Jain, Surflex: fully automatic flexible molecular docking using a molecular similarity-based search engine. Journal of medicinal chemistry. 46 (2003) 499-511. 